PROCEEDINGS OF THE

AMERICAN MATHEMATICAL SOCIETY

Volume 130, Number 1, Pages 81-84

S 0002-9939(01)06081-6

Article electronically published on April 26, 2001

\title{
ON GENERALIZED WEYL OPERATORS
}

\author{
DRAGAN S. DJORDJEVIĆ
}

(Communicated by Joseph A. Ball)

\begin{abstract}
The "generalized Weyl" operators between two Hilbert spaces are taken to be those with closed range for which the null space and that of the adjoint are of equal Hilbert space dimension. We show that products of two of these which happen to have closed range, and finite rank perturbation of these, are also generalized Weyl.
\end{abstract}

\section{INTRODUCTION}

In this paper $H, K$ and $M$ are arbitrary Hilbert spaces. We use $\mathcal{L}(H, K)$ to denote the set of all bounded operators from $H$ into $K$. Let $\operatorname{dim} L$ denote the orthogonal dimension of any closed subspace of a Hilbert space. We use $L \oplus N$ to denote the orthogonal sum of closed subspaces $L$ and $N$ of a Hilbert space. If $T \in \mathcal{L}(H, K)$, then $\mathcal{R}(T)$ is the range and $\mathcal{N}(T)$ is the kernel of $T$. It is well-known that the set of Fredholm operators from $H$ into $K$ is defined as ([6], [8])

$$
\begin{aligned}
\Phi(H, K)=\{T \in \mathcal{L}(H, K): & \mathcal{R}(T) \text { is closed, } \\
& \left.\operatorname{dim} \mathcal{N}(T)<\infty \text { and } \operatorname{dim} \mathcal{N}\left(T^{*}\right)<\infty\right\} .
\end{aligned}
$$

If $T \in \Phi(H, K)$ and $S \in \Phi(K, M)$, then $S T \in \Phi(H, M)$. For a Fredholm operator $T \in \Phi(H, K)$ the index is defined as $\operatorname{ind}(T)=\operatorname{dim} \mathcal{N}(T)-\operatorname{dim} \mathcal{N}\left(T^{*}\right)$. The class of Weyl operators from $H$ into $K$ is defined as the set of Fredholm operators of the index equal to 0 , i.e.

$$
\Phi_{0}(H, K)=\{T \in \Phi(H, K): \operatorname{ind}(T)=0\} .
$$

If $T \in \Phi(H, K)$ and $S \in \Phi(K, M)$, then the well-known index theorem states that $\operatorname{ind}(S T)=\operatorname{ind}(T)+\operatorname{ind}(S)$. Hence, if $T \in \Phi_{0}(H, K)$ and $S \in \Phi_{0}(K, M)$, then $\left.S T \in \Phi_{0}(H, M)([5], 6],[]\right)$.

In this article we introduce the class of generalized Weyl operators in the following way:

$$
\Phi_{0}^{g}(H, K)=\left\{T \in \mathcal{L}(H, K): \mathcal{R}(T) \text { is closed and } \operatorname{dim} \mathcal{N}(T)=\operatorname{dim} \mathcal{N}\left(T^{*}\right)\right\} .
$$

If $T \in \Phi_{0}^{g}(H, K)$, then $\mathcal{N}(T)$ and $\mathcal{N}\left(T^{*}\right)$ may be mutually isomorphic infinitedimensional Hilbert spaces.

Received by the editors May 17, 2000.

2000 Mathematics Subject Classification. Primary 47A53, 47A55.

Key words and phrases. Generalized Weyl operators, products of generalized Weyl operators, perturbations. 
Recently, several papers appeared concerning the generalization of Fredholm and semi-Fredholm operators, index theorem, etc. ([2], [3], [4], [9]). However, the results obtained in this article seem to be unknown and should be interesting.

It is well-known that the product of two operators with closed ranges need not to be an operator with closed range (1], [5, 12]). If $T$ and $S$ are generalized Weyl operators and $S T$ has closed range, we shall prove that $S T$ is also a generalized Weyl operator.

We use $\mathcal{F}(H, K)$ to denote the set of all finite-dimensional operators from $H$ into $K$. We shall prove that if $T$ is a generalized Weyl operator, then $T+F$ is generalized Weyl for any $F \in \mathcal{F}(H, K)$.

\section{RESUlts}

First, we shall consider products of generalized Weyl operators. Recall that a product of two Weyl operators is also a Weyl operator, but a product of two operators with closed ranges is not necessary an operator with closed range.

Theorem 1. Let $H, K$ and $M$ be arbitrary Hilbert spaces, $T \in \Phi_{0}^{g}(H, K), S \in$ $\Phi_{0}^{g}(K, M)$ and $\mathcal{R}(S T)$ is closed. Then $S T \in \Phi_{0}^{g}(H, M)$.

Proof. Consider the following matrix form of $T$ :

$$
T=\left[\begin{array}{cc}
T_{1} & 0 \\
0 & 0
\end{array}\right]:\left[\begin{array}{c}
\mathcal{R}\left(T^{*}\right) \\
\mathcal{N}(T)
\end{array}\right] \rightarrow\left[\begin{array}{c}
\mathcal{R}(T) \\
\mathcal{N}\left(T^{*}\right)
\end{array}\right]
$$

where $T_{1}: \mathcal{R}\left(T^{*}\right) \rightarrow \mathcal{R}(T)$ is invertible. We conclude that $S$ must have the matrix form

Notice that

$$
S=\left[\begin{array}{cc}
S_{1} & S_{2} \\
0 & 0
\end{array}\right]:\left[\begin{array}{c}
\mathcal{R}(T) \\
\mathcal{N}\left(T^{*}\right)
\end{array}\right] \rightarrow\left[\begin{array}{c}
\mathcal{R}(S) \\
\mathcal{N}\left(S^{*}\right)
\end{array}\right]
$$

$$
S T=\left[\begin{array}{cc}
S_{1} T_{1} & 0 \\
0 & 0
\end{array}\right]:\left[\begin{array}{c}
\mathcal{R}\left(T^{*}\right) \\
\mathcal{N}(T)
\end{array}\right] \rightarrow\left[\begin{array}{c}
\mathcal{R}(S) \\
\mathcal{N}\left(S^{*}\right)
\end{array}\right]
$$

Since $\mathcal{R}(S T)=\mathcal{R}\left(S_{1} T_{1}\right)=\mathcal{R}\left(S_{1}\right)$, we conclude that $\mathcal{R}\left(S_{1}\right)$ is closed. We can write $\mathcal{R}(S)=\mathcal{R}\left(S_{1}\right) \oplus \mathcal{N}\left(S_{1}^{*}\right)$. Let $N=S_{2}^{-1}\left(\mathcal{R}\left(S_{1}\right)\right)$. Then $\mathcal{N}\left(S_{2}\right) \subset N$ and $\mathcal{N}\left(T^{*}\right)=N \oplus N^{\perp}$. The set $S_{2}\left(N^{\perp}\right)$ is the subspace of $\mathcal{R}(S)$ linearly independent modulo $\mathcal{R}\left(S_{1}\right)$ and $\mathcal{R}(S)=\mathcal{R}\left(S_{1}\right)+\mathcal{R}\left(S_{2}\right)$. Define an operator $S_{3} \in$ $\mathcal{L}\left(\mathcal{N}\left(T^{*}\right), \mathcal{R}(S)\right)$ in the following way:

$$
S_{3} u= \begin{cases}0, & u \in N, \\ S_{2} u, & u \in N^{\perp} .\end{cases}
$$

Now $\mathcal{R}\left(S_{3}\right)=S_{2}\left(N^{\perp}\right), \mathcal{R}\left(S_{3}\right) \cap \mathcal{R}\left(S_{1}\right)=\{0\}$ and $\mathcal{R}(S)=\mathcal{R}\left(S_{1}\right)+\mathcal{R}\left(S_{3}\right)$. According to the well-known Kato theorem ([11]), we know that $\mathcal{R}\left(S_{3}\right)=S_{2}\left(N^{\perp}\right)$ is closed. We conclude $S_{2}\left(N^{\perp}\right) \cong \mathcal{R}(S) / \mathcal{R}\left(S_{1}\right) \stackrel{\cong}{=} \mathcal{N}\left(S_{1}^{*}\right)$; hence $\operatorname{dim} N^{\perp}=\operatorname{dim} \mathcal{N}\left(S_{1}^{*}\right)$.

On the other hand,

$$
\begin{aligned}
\mathcal{N}(S T) & =\mathcal{N}(T) \oplus\left\{x \in \mathcal{R}\left(T^{*}\right): S_{1} T_{1} x=0\right\}=\mathcal{N}(T) \oplus T_{1}^{-1}\left(\mathcal{N}\left(S_{1}\right)\right) \\
& \simeq \mathcal{N}\left(T^{*}\right) \oplus \mathcal{N}\left(S_{1}\right)=N \oplus N^{\perp} \oplus \mathcal{N}\left(S_{1}\right) .
\end{aligned}
$$

Since

$$
\mathcal{N}\left((S T)^{*}\right)=\mathcal{N}\left(S^{*}\right) \oplus \mathcal{N}\left(S_{1}^{*}\right) \stackrel{\sim}{N}(S) \oplus \mathcal{N}\left(S_{1}^{*}\right)
$$


and $\operatorname{dim} N^{\perp}=\operatorname{dim} \mathcal{N}\left(S_{1}^{*}\right)$, we only need to prove that $\mathcal{N}(S) \stackrel{\cong}{=} \oplus \mathcal{N}\left(S_{1}\right)$. Notice that if $x \in \mathcal{R}(T)$ and $y \in \mathcal{N}\left(T^{*}\right)$, then $z=\left[\begin{array}{l}x \\ y\end{array}\right] \in \mathcal{N}(S)$ if and only if $S_{1} x=-S_{2} y$. Hence, $S_{1} x=0$ if and only if $S_{2} y=0$, implying $\mathcal{N}\left(S_{1}\right) \oplus \mathcal{N}\left(S_{2}\right) \subset \mathcal{N}(S)$. Let $N=\mathcal{N}\left(S_{2}\right) \oplus L$. If $y \in L$, then there exists the unique $x \in \mathcal{R}\left(S_{1}^{*}\right)$ such that $S_{1} x=-S_{2} y$. We conclude that

$$
\mathcal{N}\left(S_{1}\right) \oplus N=\mathcal{N}\left(S_{1}\right) \oplus \mathcal{N}\left(S_{2}\right) \oplus L \cong \mathcal{N}(S) .
$$

Thus, the proof is completed.

If $S, T$ and $S T$ have closed ranges, then Harte's ghost theorem ([9]) states that

$$
\mathcal{N}(T) \times \mathcal{N}(S) \times M / \mathcal{R}(S T) \cong \mathcal{N}(S T) \times K / \mathcal{R}(T) \times M / \mathcal{R}(S) .
$$

Notice that our Theorem 1 does not follow from this ghost theorem. For example, it might be possible that $\mathcal{N}(T) \times \mathcal{N}(S)$ and $K / \mathcal{R}(T) \times M / \mathcal{R}(S)$ are mutually isomorphic infinite-dimensional Hilbert spaces, but $\operatorname{dim} \mathcal{N}(S T)=1$ and $\operatorname{dim} M / \mathcal{R}(S T)=2$.

It is also interesting to consider a perturbation result for generalized Weyl operators by a finite-dimensional operator. We can prove the following statement.

Theorem 2. Let $H, K$ be arbitrary Hilbert spaces, $T \in \Phi_{0}^{g}(H, K)$ and $F \in \mathcal{F}(H, K)$. Then $T+F \in \Phi_{0}^{g}(H, K)$.

Proof. The result is already known if $\mathcal{N}(T)$ and $\mathcal{N}\left(T^{*}\right)$ are mutually isomorphic finite-dimensional subspaces. Hence, assume that $\mathcal{N}(T)$ and $\mathcal{N}\left(T^{*}\right)$ are mutually isomorphic infinite-dimensional subspaces, $\mathcal{R}(T)$ is closed and $F \in \mathcal{F}(H, K)$.

We can write $H=\mathcal{N}(F) \oplus \mathcal{N}(F)^{\perp}$, where $\operatorname{dim} H / \mathcal{N}(F)=\operatorname{dim} \mathcal{N}(F)^{\perp}=$ $\operatorname{dim} \mathcal{R}(F)<\infty$. Now we have

$$
T(\mathcal{N}(F)) \subset(T+F)(\mathcal{N}(F))+(T+F)\left(\mathcal{N}(F)^{\perp}\right)=\mathcal{R}(T+F) \subset \mathcal{R}(T)+\mathcal{R}(F) .
$$

Since $\operatorname{dim} \mathcal{R}(T) / T(\mathcal{N}(F))<\infty$, we conclude that $\mathcal{R}(T+F)$ and $\mathcal{R}(T)$ may differ for a finite-dimensional subspace, so we get that $\mathcal{R}(T+F)$ is closed. Since $\mathcal{N}\left(T^{*}\right)$ is infinite-dimensional, we also find

$$
\operatorname{dim} \mathcal{N}(T+F)^{*}=\operatorname{dim} K / \mathcal{R}(T+F)=\operatorname{dim} K / \mathcal{R}(T)=\operatorname{dim} \mathcal{N}\left(T^{*}\right) .
$$

We can also write $H=\mathcal{N}(T) \oplus \mathcal{N}(T)^{\perp}$ and denote $W=\left\{v \in \mathcal{N}(T)^{\perp}: T v \in\right.$ $\mathcal{R}(F)\}$. Let $x \in \mathcal{N}(T+F)$ and $x=u+v$, where $u \in \mathcal{N}(T)$ and $v \in \mathcal{N}(T)^{\perp}$. Then $T v=-F x \in \mathcal{R}(F)$. We conclude $\mathcal{N}(T+F) \subset \mathcal{N}(T)+W$ and $\operatorname{dim} \mathcal{N}(T+F) \leq$ $\operatorname{dim} \mathcal{N}(T)+\operatorname{dim} W=\operatorname{dim} \mathcal{N}(T)+\operatorname{dim} \mathcal{R}(F)=\operatorname{dim} \mathcal{N}(T)$. In the same way we can prove that $\operatorname{dim} \mathcal{N}(T)=\operatorname{dim} \mathcal{N}(T+F)+(-F)) \leq \mathcal{N}(T+F)$.

Hence, $\operatorname{dim} \mathcal{N}(T+F)=\operatorname{dim} \mathcal{N}(T+F)^{*}$ and the proof is completed.

The previous result is an extension of the well-known result concerning the perturbation of an ordinary Weyl operator by a finite-dimensional operator. However, we cannot expect that the perturbation result by a compact operator may hold. Precisely, in [5] it is shown that if $\mathcal{R}(T)$ is closed, $\mathcal{N}(T)$ and $\mathcal{R}(T)$ are both infinitedimensional, then there exists a compact operator $C$, such that $\mathcal{R}(T+\lambda C)$ is not closed for any $\lambda \in \mathbb{C} \backslash 0$. It also follows that $\Phi_{0}^{g}(H, K)$, in general, is not an open subset of $\mathcal{L}(H, K)$. 
Remarks. The case $H=K=M$ can be considered in a more general context, and in this case results of this paper are already known. If $\mathcal{A}$ is a Banach algebra (or, more generally, an additive category), then Harte [7] defined relatively Weyl elements as the set of all elements $a \in \mathcal{A}$ such that $a \in a \mathcal{A}^{-1} a$. Here $\mathcal{A}^{-1}$ denotes the set of all invertible elements of $\mathcal{A}$. If $\mathcal{A}=\mathcal{L}(\mathcal{H})$, then the set of relatively Weyl elements coincides with our set of generalized Weyl operators. In this case our Theorem 1 follows from [7, Theorem 1], and our Theorem 2 is proved in [10. Theorem 7].

Moreover, it would be interesting to consider the same problems in the case when $T$ is a bounded operator from a Banach space $X$ into a Banach space $Y$. We say that $T$ is generalized Weyl if $\mathcal{R}(T)$ is closed, and $\mathcal{N}(T)$ and $Y / \mathcal{R}(T)$ are mutually isomorphic Banach spaces. This case is not completely covered by 7$]$ and 10$]$. It is also not clear that our proofs of Theorem 1 and Theorem 2 are valid in Banach spaces.

\section{ACKNOWLEDGEMENT}

I am grateful to Professor Laura Burlando for helpful correspondence and suggestions. I am also grateful to the Referee for helpful comments and suggestions concerning the paper

\section{REFERENCES}

[1] R. H. Bouldin, The product of operators with closed range, Tôhoku Math. J. 25 (1973), 359-363. MR 48:4768

[2] R. H. Bouldin, Generalizations of semi-Fredholm operators, Proc. Amer. Math. Soc. 123 (1995), 3757-3764. MR 96i:47020

[3] L. Burlando, Approximation by semi-Fredholm and semi- $\alpha$-Fredholm operators in Hilbert spaces of arbitrary dimension, Acta Sci. Math. (Szeged) 65 (1999), 217-275. MR 2000j:47020

[4] L. Burlando, On the weighted reduced minimum modulus, Proceeding of the $17^{\text {th }}$ Conference in Operator Theory (Timisoara, 1998) (2000), 47-66. CMP 2000:17

[5] S. R. Caradus, Generalized Inverses and Operator Theory, Queen's paper in pure and applied mathematics, Queen's University, Kingston, Ontario, 1978.

[6] S. R. Caradus, W. E. Pfaffenberger and B. Yood, Calkin Algebras and Algebras of Operators on Banach Spaces, Marcel Dekker, New York, 1974.

[7] R. E. Harte, Regular boundary elements, Proc. Amer. Math. Soc. 99 (1987), 328-330. MR 88d:46088

[8] R. E. Harte, Invertibility and Singularity for Bounded Linear Operators, New York, Marcel Dekker, 1988. MR 89d:47001

[9] R. E. Harte, The ghost of an index theorem, Proc. Amer. Math. Soc. 106 (1989), 1031-1033. MR 92j: 47029

[10] R. E. Harte and H. Raubenheimer, Fredholm, Weyl and Browder theory III, Proc. R. Ir. Acad. 95A (1995), 11-16. MR 96i:47002

[11] T. Kato, Perturbation Theory for Linear Operators, Springer-Verlag, Berlin-Heidelberg-New York, 1976. MR 53:11389

[12] J. J. Koliha, The product of relatively regular operators, Comm. Math. Univ. Carolinae 16, 3 (1975), 531-539. MR 52:1373

Department of Mathematics, Faculty of Sciences and Mathematics, University of Niš, Ćirila i MetodiJa 218000 Niš, Yugoslavia

E-mail address: dragan@pmf.pmf.ni.ac.yu

E-mail address: ganedj@eunet.yu 